\section{LA GRANJA:}

REVISTA DE

CIENCIAS DE LA VIDA

pISSN:1390-3799; eISSN:1390-8596

http:/ / doi.org/10.17163/lgr.n27.2018.08
Artículo ciertífico / Scientific paper

REMEDIACIÓN

\title{
TRATAMIENTO DE AGUAS RESIDUALES DE CARGAS INDUSTRIALES CON OXIDACIÓN AVANZADA EN SISTEMAS CONVENCIONALES
}

\author{
WASTEWATER TREATMENT OF INDUSTRIAL LOADS WITH ADVANCED \\ OXIDATION IN CONVENTIONAL SYSTEMS
}

\section{Renato Gabriel Sánchez Proaño ${ }^{1,2}$, Katty J. García Gualoto ${ }^{2}$}

\author{
${ }^{1}$ Grupo de Investigación en Ciencias Ambientales, GRICAM, Ingeniería ambiental; Universidad Politécnica Salesiana del Ecua- \\ dor, sede Quito: Av. Morán Valverde y Rumichaca s/n. \\ ${ }^{2}$ Carrera de Ingeniería Ambiental. Universidad Politécnica Salesiana, Quito, Ecuador, Rumichaca y Morán Valverde s/n-Quito, \\ Tel. (+593) 2 3962900/3962800.
}

*Autor para correspondencia: rsanchezp@ups.edu.ec

\begin{abstract}
Resumen
En las regiones rurales de América Latina los sistemas de tratamiento de agua residuales se basan en tecnologías convencionales como las fosas sépticas. Estos sistemas de tratamiento no soportan índices de biodegradabilidad menores a 0,4 medidos en forma de $\mathrm{DBO}_{5} / \mathrm{DQO}$, valores que se obtienen cuando en el agua se encuentran contaminantes refractarios; que a su vez se los asocia en la mayoría de los casos con la intromisión de agua residual industrial. En estos casos los sistemas de tratamiento convencionales no cumple las normativas para los cuales fueron diseñados; y por tanto se pierde la inversión pública para construirlos. Es por esto que en la presente investigación se elevó el índice de biodegradabilidad de este tipo de efluentes, para lo cual se usó agua residual doméstica mezclada con lixiviado y efluente industrial obtenida de la quebrada Chasinato en la provincia de Tungurahua en Ecuador, en la cual se obtuvo un índice de biodegradabilidad de 0,22 en $\mathrm{DBO}_{5} / \mathrm{DQO}$. A este efluente se le aplicó un proceso de oxidación avanzada de Fenton modificado, con lo cual se incrementó el índice de biodegradabilidad y se evitó reducir el $\mathrm{pH}$, ya que en grandes volúmenes esto resulta muy costoso. Las concentraciones óptimas de reactivos fueron de $\mathrm{FeSO}_{4} / \mathrm{H}_{2} \mathrm{O}_{2}=1$ y $\mathrm{C}_{6} \mathrm{H}_{8} \mathrm{O}_{7} / \mathrm{H}_{2} \mathrm{O}_{2}=2$; con la colocación de $10 \mathrm{mg} / \mathrm{L}$ de $\mathrm{H}_{2} \mathrm{O}_{2}$, con lo cual se aumentó el índice de biodegradabilidad a un valor de 0,46 que es un valor satisfactorio para tratarse en sistemas convencionales como son las fosas sépticas; además de una reducción de la $\mathrm{DBO}_{5}$ y DQO en eficiencias de $12,54 \% ; 44,4 \%$ respectivamente.

Palabras claves: Oxidación avanzada, Fenton modificado, contaminantes refractarios, tratamiento de lixiviados, fosa séptica.
\end{abstract}




\begin{abstract}
In the rural regions of Latin America, wastewater treatment systems are based on conventional technologies such as the septic tanks. These treatment systems do not support biodegradability indexes lower than 0,4 measured in the form of $\mathrm{BOD}_{5} / \mathrm{COD}$, values obtained when refractory contaminants are found in water; which in turn are associated in most cases with the intrusion of industrial wastewater. In these cases, the conventional treatment systems do not meet the regulations for which they were designed; and therefore public investment is lost to build them. This is why in the present investigation the biodegradability index of this type of effluents was increased, for which domestic wastewater mixed with leachate and industrial effluent obtained from the Chasinato creek in the Tungurahua province of Ecuador was used, in which a biodegradability index of 0,22 was obtained in $\mathrm{BOD}_{5} / \mathrm{COD}$. An advanced oxidation process of modified Fenton was applied to this effluent, which increased the biodegradability index and avoided reducing the $\mathrm{pH}$, since in large volumes this is very expensive. The optimal concentrations of reagents were $\mathrm{FeSO}_{4} / \mathrm{H}_{2} \mathrm{O}_{2}=1$ and $\mathrm{C}_{6} \mathrm{H}_{8} \mathrm{O}_{7} / \mathrm{H}_{2} \mathrm{O}_{2}=2$; with the addition of $10 \mathrm{mg} / \mathrm{L}$ of $\mathrm{H}_{2} \mathrm{O}_{2}$, which increased the biodegradability index to a value of 0,46 , which is a satisfactory value to be treated in conventional systems such as septic tanks; in addition to a reduction of $\mathrm{BOD}_{5}$ and $\mathrm{COD}$ in efficiencies of $12.54 \% ; 44.4 \%$ respectively.
\end{abstract}

Keywords: Advanced oxidation, modified Fenton, refractory contaminants, leachate treatment, septic tank.

Forma sugerida de citar: Sánchez Proaño, R. G. y García Gualoto, K. J. 2018. Tratamiento de aguas residuales de cargas industriales con oxidación avanzada en sistemas convencionales. La Granja: Revista de Ciencias de la Vida. Vol. 27(1):103-111. http://doi.org/10.17163/lgr.n27.2018. 08 . 


\section{Introducción}

En las regiones rurales de América Latina y el Caribe, la cobertura de sistemas de alcantarillado fue del $64 \%$ para el año 2015 (Lentini, 2015), llegándose a tratar el agua residual en un $34 \%$ (UNESCO, 2017). La mayoría de estos sistemas de tratamiento son plantas convencionales construidas en las etapas finales de los sistemas de alcantarillado, los cuales se ubican antes de una descarga a cuerpos de agua. Estos sistemas de tratamiento se basan en tecnologías de remoción de baja carga contaminante y bajo costo de construcción y operación. La tecnología más usada es la fosa séptica, construida generalmente de concreto o plástico (Jouravlev, 2004).

Las fosas sépticas se usan como sistemas de tratamiento primario (Metcalf y Eddy, 2003) que remueven aceites, grasas y sólidos suspendidos en un $50 \%$ (Méndez et al., 2012), convirtiendo los sólidos suspendidos volátiles a los fijos. La eficiencia en remoción de la demanda bioquímica de oxígeno (DBO5), sólidos suspendidos totales (SST), y coliformes totales van desde menos del 50\% hasta el $80 \%$ en función de la temperatura (Lucho-Constantino et al., 2015).

El índice de biodegradabilidad es un factor de influencia en la eficiencia de una fosa séptica, que debe ser mayor a 0,4 calculado con el factor $\mathrm{DBO}_{5} / \mathrm{DQO}$ (Ortiz de Zárate y Aguila Apodaca, 1997), lo cual no se cumple cuando las aguas residuales presentan contaminantes refractarios, que están relacionados con la mezcla de las aguas residuales domésticas y aguas residuales industriales sin tratamiento. Estos contaminantes se incrementan en las aguas residuales de zonas rurales por el aumento en el uso de productos químicos en los hogares, la industria doméstica u otras actividades productivas. Entre los contaminantes refractarios presentes en el agua residual doméstica están pesticidas, medicamentos, compuestos de aseo personal, residuos de industria doméstica, entre otros (Gil et al., 2013); y cuando sus concentraciones son muy altas, se reducen sus índices de biodegradabilidad hasta niveles en los que las eficiencias de las plantas de tratamiento convencionales no llegan a cumplir las normativas de descarga, convirtiéndose en infraestructura inservible (English, 2010).

Es por esto que en la presente investigación se propone el incremento de una etapa de tratamiento avanzado de las aguas residuales al inicio de los sistemas de convencionales con fosa séptica, para aumentar la biodegradabilidad de las aguas y permitir que el tratamiento posterior trate el agua residual con el índice de biodegradabilidad modificado.

El tratamiento de oxidación avanzada fue descrito inicialmente por Glaze, Kang y Chapin (1987), basándose en la generación de oxidantes fuertes como los radicales $\cdot \mathrm{OH}$ para la degradación de compuestos solubles no biodegradables presentes en el agua residual. El proceso de oxidación avanzada escogido es el método Fenton, el cual forma radicales -OH según la siguiente ecuación (Somich, Muldoon y Kearney, 1990; Martiínez-Huitle et al., 2008):

$$
\mathrm{Fe}_{2+}+\mathrm{H}_{2} \mathrm{O}_{2} \rightarrow \mathrm{Fe}_{3+}+\cdot \mathrm{OH}+\mathrm{OH}^{-}
$$

Como se muestra, un reactivo en el proceso Fenton es el peróxido de hidrógeno $\left(\mathrm{H}_{2} \mathrm{O}_{2}\right)$, pero este por sí solo no oxida a ciertos contaminantes refractarios; requiriendo la adición de sales de hierro para la formación de radicales hidroxilo $\cdot \mathrm{OH}$. Con la presencia de $\cdot \mathrm{OH}$, oxidarán de forma no específica los compuestos orgánicos a altas velocidades (Pawar y Gawande, 2015).

En una reacción Fenton el pH se controla en un rango de 3 a 5 (Pawar y Gawande, 2015), pero al tener agua residual con $\mathrm{pH}$ cercanos a 7 , se volvería muy costosa la disminución y posterior aumento del $\mathrm{pH}$. Es por esto que se ha optado por la agregación de un reactivo adicional, un quelante para inducir a la formación de un complejo; y de esta manera producir la reacción Fenton a un $\mathrm{pH}$ neutro o cercano a este valor (Isch Zambrano, 2016).

Uno de los compuestos más usados para la formación de quelatos es el EDTA, sin embargo, los coloides formados por este reactivo son muy estables, y resultaría compleja su degradación en los sistemas biológicos subsecuentes (Oviedo y Rodríguez, 2003). Es por esto que se usará ácido cítrico el cual es considerado como un agente quelante que puede secuestrar hierro en presencia de amoniaco, además de poseer numerosas aplicaciones por su capacidad para formar complejos solubles (REPAMAR, 1998).

Para la presente investigación, como muestra de agua residual se ha elegido una descarga en la quebrada de "Chasinato" en el cantón Ambato, provincia de Tungurahua en Ecuador, cuya ubicación se muestra en la Figura 1. Esta descarga de agua posee baja biodegradabilidad debido a que el agua residual doméstica en las cotas superiores se mezclan con lixiviado del relleno sanitario de la ciudad de Ambato, aguas residuales del Parque Industrial de la ciudad, un matadero de animales, entre otros asentamientos domiciliares e industriales. 


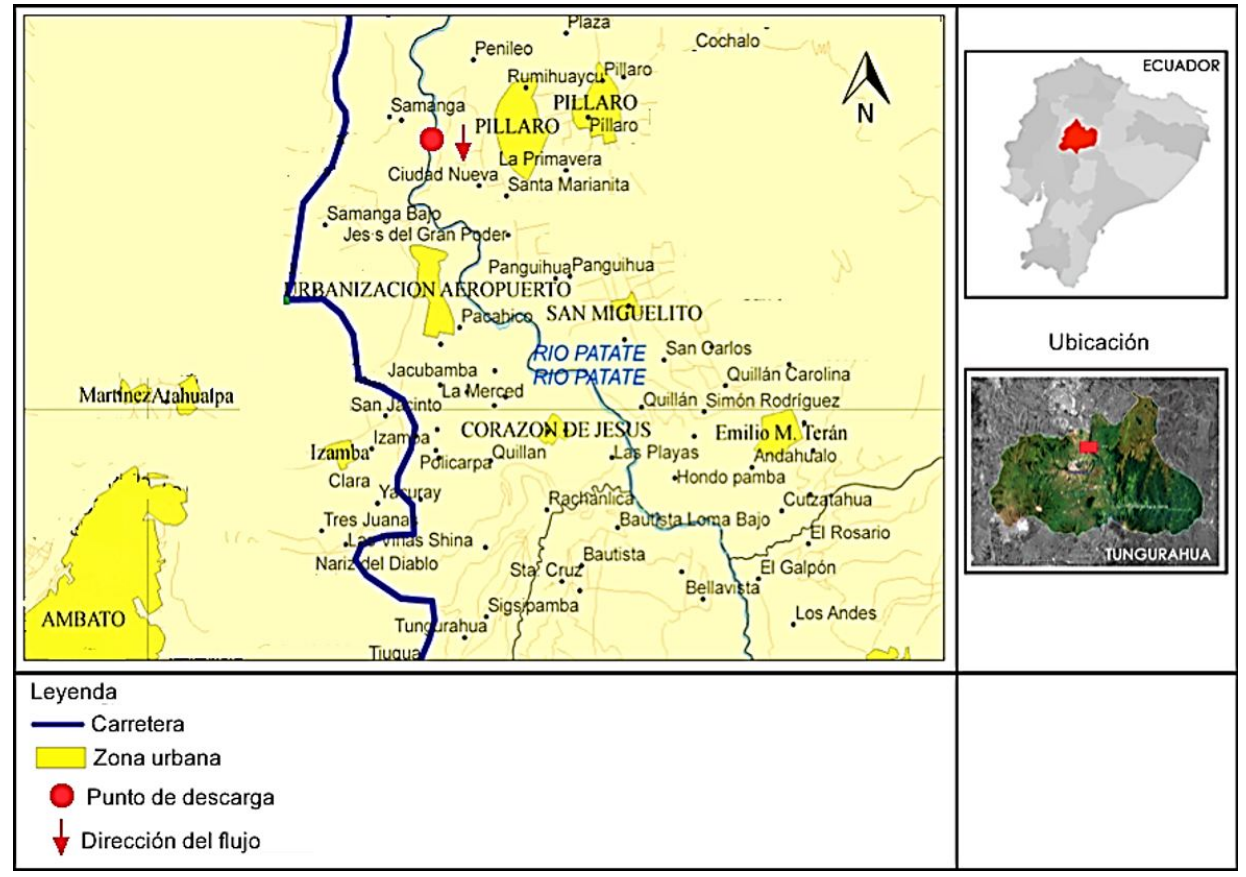

Figura 1. Ubicación del punto de muestreo del agua residual doméstica combinada con agua industrial y lixiviado de relleno sanitario (MTOP, 2010)

Una vez que el agua residual del cuerpo de agua del que se tomó la muestra para la presente investigación es vertida hacia la quebrada Chasinato, esta desemboca en el río Cutuchi; que aguas abajo es usada para regar cultivos en las zonas Quillán Playas, Chiquicha, Bellavista, Los Andes, etc., hasta llegar al río Pastaza.

\section{Materiales y métodos}

\subsection{Agua residual}

Las muestras de agua residual se obtuvieron en la quebrada Chasinato en marzo del año 2016; para el análisis se tomaron 40 litros de agua residual en dos recipientes plásticos de 20 litros sellados, transportados al laboratorio para ser analizados.

\subsection{Protocolos de análisis}

Los protocolos de análisis fueron realizados según el Standard Methods (Pawlowski, 1994) para los parámetros de Demanda química de oxígeno (DQO), con la técnica de reflujo cerrado y volumetría (Standard Methods 5220C), la Demanda bioquímica de oxígeno $\left(\mathrm{DBO}_{5}\right)$ con la técnica de incubación en 5 días y uso de oxímetro (Standard Methods 4500), $\mathrm{pH}$ y conductividad eléctrica.

\subsection{Reacción Fenton}

El procedimiento elegido para la descontaminación experimental es la reacción de oxidación avanzada tipo Fenton modificado. La reacción Fenton fue realizada a $20^{\circ} \mathrm{C}$ y a la presión atmosférica de la ciudad de Quito (546 mm Hg).

Las condiciones óptimas del reactivo Fenton se obtienen a valores ácidos de $\mathrm{pH}$ (Méndez et al., 2010). La reducción del pH en los efluentes de agua residual doméstica resulta muy costosa debido a los grandes caudales a tratarse; por lo que, como se señaló antes, se ha elegido una técnica de Fenton modificado mediante el uso de un agente quelante, escogiéndose el ácido cítrico, ya que este puede formar quelatos de $\mathrm{Fe}^{3+}$ más estables que con $\mathrm{Fe}^{2+}$. La actividad de los quelatos del $\mathrm{Fe}^{3+}$ formados con agentes quelantes es baja para disociar el $\mathrm{H} 2 \mathrm{O} 2$, formando así complejos menos estables (Seol y I., 2008); los cuales se pueden degradar de mejor forma en la siguiente etapa de la planta de tratamiento de aguas residuales que es el sistema biológico. 
La estabilidad de los quelatos es inversamente proporcional al incremento de las constantes de estabilidad (log k), los cuales son de 7,49; 9,4 y 11,85 para los ácidos tartárico, oxálico y cítrico respectivamente (Furia, 1973). De esta manera los quelatos fuertes del ácido cítrico- $\mathrm{Fe}^{3+}$ al dar una baja actividad de $\mathrm{Fe}^{3+}$ inhiben a las especies de hierro de volver a reducirse a $\mathrm{Fe}^{2+}$ cortando el ciclo catalítico de la reacción Fenton (Seol y I., 2008). La presencia de nitrógeno en forma de amoniaco por la contribución del agua residual doméstica mejora la capacidad del ácido cítrico de secuestrar hierro y formar complejos solubles (REPAMAR, 1998), esto puede ocurrir en las fosas séptica bien construidas en donde se generen procesos anaerobios.

Para el tratamiento experimental se procedió a realizar varias mezclas de $\mathrm{H}_{2} \mathrm{O}_{2}, \mathrm{FeSO}_{4}$ y ácido cítrico $\left(\mathrm{C}_{6} \mathrm{H}_{8} \mathrm{O}_{7}\right)$ en las proporciones indicadas en la
Tabla 1 (Zazouli et al., 2012; Mashal et al., 2012; Barbusiński y Pieczykolan, 2010). No se modificó el pH del agua residual, por lo que este parámetro para todas las mezclas realizadas antes de la reacción Fenton fue de 7,21. Como tratamiento inicial a las mezclas Fenton se realizó una decantación del agua residual durante una hora para remover los sólidos suspendidos. Los ensayos se realizaron por duplicado mezclando el agua residual, $\mathrm{FeSO}_{4}, \mathrm{H}_{2} \mathrm{O}_{2}$ y el $\mathrm{C}_{6} \mathrm{H}_{8} \mathrm{O}_{7}$ a $100 \mathrm{rpm}$ durante un minuto y posteriormente a $30 \mathrm{rpm}$ durante cuatro minutos en un mezclador para prueba de jarras (Figura 2). Los análisis de $\mathrm{DBO}_{5}$ y DQO se realizaron una hora después de haber parado la mezcla mecánica, ya que este método requiere tiempos de reacción relativamente cortos y utiliza reactivos fáciles de manejar (Bautista et al., 2008).

Tabla 1. Mezclas de $\mathrm{H}_{2} \mathrm{O}_{2}, \mathrm{FeSO}_{4} \mathrm{y}_{6} \mathrm{H}_{8} \mathrm{O}_{7}$ para reacción Fenton modificado.

\begin{tabular}{cccc}
\hline \multirow{2}{*}{ Mezcla } & \multicolumn{3}{c}{ Reactivo Fenton modificado (mg/L) } \\
\cline { 2 - 4 } & $\mathbf{H}_{2} \mathbf{O}_{2}$ & FeSO $_{4}$ & $\mathbf{C}_{6} \mathbf{H}_{8} \mathbf{O}_{7}$ \\
\hline $\mathbf{1}$ & 10 & 10 & 10 \\
$\mathbf{2}$ & 10 & 10 & 20 \\
$\mathbf{3}$ & 10 & 20 & 10 \\
$\mathbf{4}$ & 10 & 20 & 20 \\
$\mathbf{5}$ & 20 & 10 & 10 \\
$\mathbf{6}$ & 20 & 10 & 20 \\
\hline
\end{tabular}

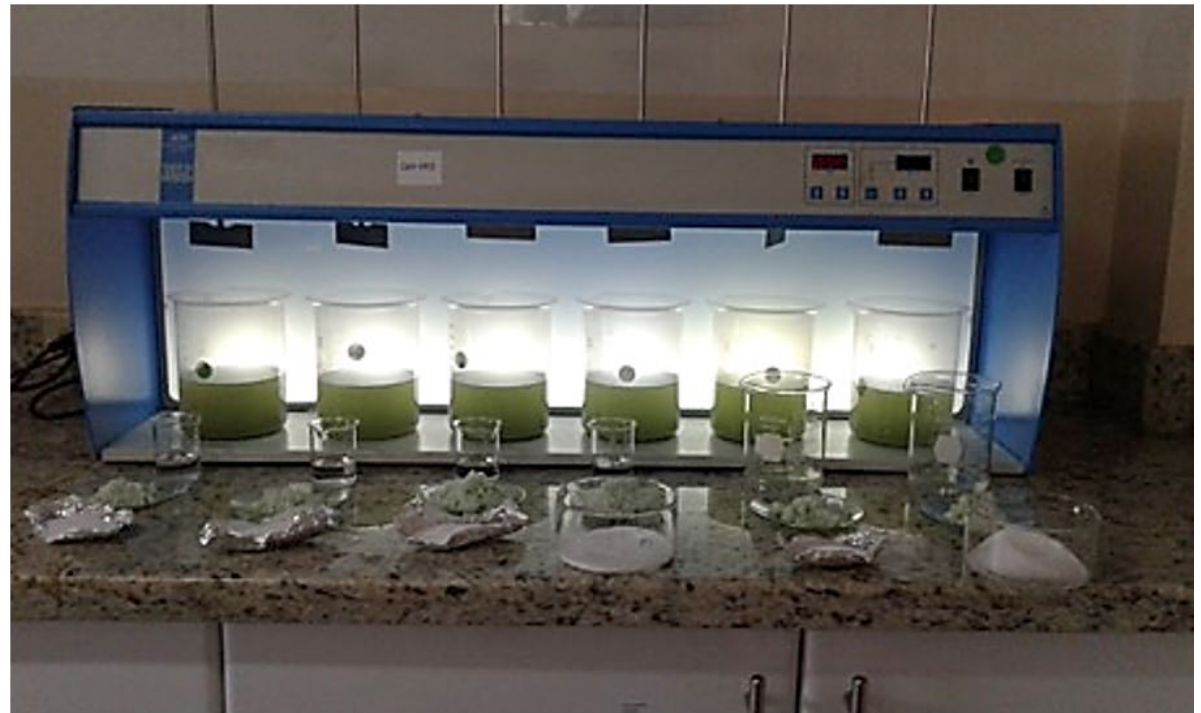

Figura 2. Fotografía de una prueba de jarras realizada con distintas concentraciones de los reactivos de Fenton modificado: $\mathrm{H}_{2} \mathrm{O}_{2}$, $\mathrm{FeSO}_{4}$ y $\mathrm{C}_{6} \mathrm{H}_{8} \mathrm{O}_{7}$; realizado al agua residual de la muestra. 


\section{Resultados}

Los resultados de los análisis iniciales de calidad de agua se los muestra en la Tabla 2.

Tabla 2. Resultados de calidad inicial de agua residual

\begin{tabular}{cccc}
\hline pH & DQO & DBO $_{5}$ & Relación DBO $_{5} /$ DQO \\
\hline 7,21 & $11750 \mathrm{mg} / \mathrm{L}$ & $2606 \mathrm{mg} / \mathrm{L}$ & 0,22 \\
\hline
\end{tabular}

Los resultados de cada mezcla se muestran en la Tabla 3. Como se esperaba, los valores de $\mathrm{DBO}_{5} \mathrm{y}$ DQO siguen siendo elevados. Sin embargo, los valores de biodegradabilidad de la materia orgánica se incrementaron, siendo el valor óptimo en una mezcla 6 con $20 \mathrm{mg} / \mathrm{L}$ de $\mathrm{H}_{2} \mathrm{O}_{2}, 10 \mathrm{mg} / \mathrm{L}$ de $\mathrm{FeSO}_{4}$ y 20 $\mathrm{mg} / \mathrm{L}$ de $\mathrm{C}_{6} \mathrm{H}_{8} \mathrm{O}_{7}$ a pesar de tener un valor de $\mathrm{pH}$ no óptimo para la reacción Fenton clásica. Se debe señalar que en las mezclas 3 y 4 se observaron precipitados de óxidos de hierro, debido posiblemente a una co-precipitación acompañada a una disminución en la remoción de la DQO; por lo que no se realizaron mezclas en mayores proporciones.

Tabla 3. DQO, $\mathrm{DBO}_{5}$ e índice de biodegradabilidad de las mezclas de $\mathrm{H}_{2} \mathrm{O}_{2}, \mathrm{FeSO}_{4}$ y $\mathrm{C}_{6} \mathrm{H}_{8} \mathrm{O}_{7}$.

\begin{tabular}{cccc}
\hline Mezcla & DQO (mg/L) & DBO $_{5}(\mathbf{m g} / \mathrm{L})$ & DBO $_{5} / \mathrm{DQO}$ \\
\hline $\mathbf{1}$ & 7598 & 2279 & 0,3 \\
$\mathbf{2}$ & 6536 & 3007 & 0,46 \\
$\mathbf{3}$ & 11331 & 4079 & 0,36 \\
$\mathbf{4}$ & 11532 & 5420 & 0,47 \\
$\mathbf{5}$ & 8257 & 3055 & 0,37 \\
$\mathbf{6}$ & 9716 & 4761 & 0,49 \\
\hline
\end{tabular}

\section{Discusión}

Los resultados obtenidos muestran que la reacción Fenton mejora la relación de biodegradabilidad hasta que se pueda lograr un tratamiento en un sistema convencional; pero, la reacción Fenton modificado por sí misma no alcanza la reducción de la $\mathrm{DBO}_{5}$ y la DQO para estar debajo de la normativa ecuatoriana vigente a la fecha; la cual, para vertidos en cuerpos de agua dulce señala valores máximos de $100 \mathrm{mg} / \mathrm{L}$ de $\mathrm{DBO}_{5}$ y $200 \mathrm{mg} / \mathrm{L}$ para DQO (MAE, 2015). La remoción máxima de DQO se da en la mezcla 2, cuya eficiencia es de $44,4 \%$, la remoción máxima de $\mathrm{DBO}_{5}$ en $12,54 \%$ y el aumento óptimo de la biodegradabilidad en la mezcla 6, llegando a un índice de 0,49 .

Para todas las demás mezclas existe un incremento de la $\mathrm{DBO}_{5}$ en función de una disminución del DQO, debido a una descomposición y reorga- nización de las estructuras en las moléculas orgánicas desde compuestos refractarios a compuestos más biodegradables. En este caso particular, el obtener mayores eficiencias en la remoción de la DQO se debe probablemente a que en el agua residual existen compuestos orgánicos no biodegradables preferentes para la reacción Fenton, similar a lo obtenido en estudios realizados con lixiviados maduros (Lopes de Morais y Peralta Zamora, 2005).

El agua residual doméstica con carga industrial puede ingresar a la planta de tratamiento de aguas residuales con una etapa de oxidación de Fenton modificado al inicio del sistema; que con los datos tomados en campo ingresarían con una DQO y $\mathrm{DBO}_{5}$ de $11750 \mathrm{mg} / \mathrm{L}$ y $2606 \mathrm{mg} / \mathrm{L}$ respectivamente; y al pasar por la etapa de oxidación de Fenton modificado estos datos disminuirían a una DQO y $\mathrm{DBO}_{5}$ de $6536 \mathrm{mg} / \mathrm{L}$ y $3007 \mathrm{mg} / \mathrm{L}$ respectivamente (Figura 3). 


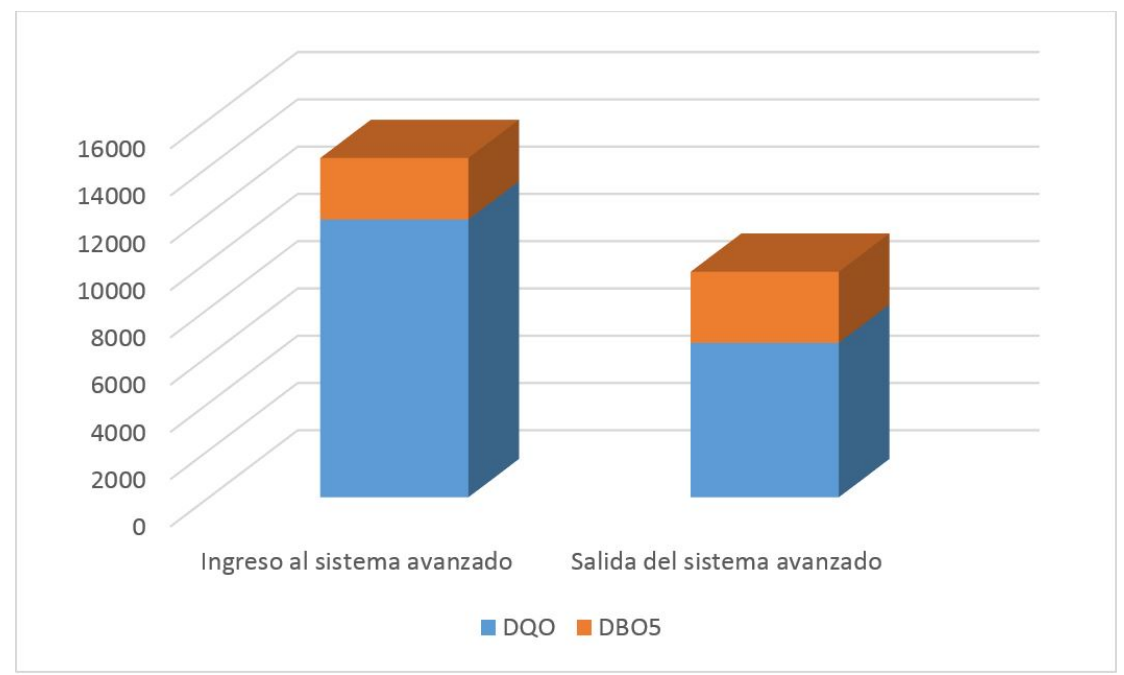

Figura 3. Gráfico de la disminución de $\mathrm{DQO}$, aumento de la $\mathrm{DBO}_{5}$ medidas en $\mathrm{mg} / \mathrm{L}$ en la concentración óptima de reactivo Fenton modificado.

En resumen, los resultados del presente trabajo demuestran la idoneidad del proceso Fenton para mejorar el índice de biodegradabilidad del agua residual doméstica con carga industrial. Los resultados demuestran que las aguas residuales domésticas mezcladas con aguas industriales y de lixiviados presentan relaciones de $\mathrm{DBO}_{5} / \mathrm{DQO}$ bajas; por lo que el proceso de oxidación avanzada no debe realizarse como tratamiento final a un sistema de tratamiento biológico; sino por el contrario, previo a un tratamiento aerobio o anaerobio (Lopes de Morais y Peralta Zamora, 2005). La ubicación del sistema de tratamiento de oxidación avanzada estará entre el sistema de tratamiento físico y el tratamiento biológico, como lo indica la Figura 4; con esto se evita el gasto de reactivos para la oxidación de materia sólida no suspendida y que es separada en el sistema de tratamiento físico de tamizado y desarenado.

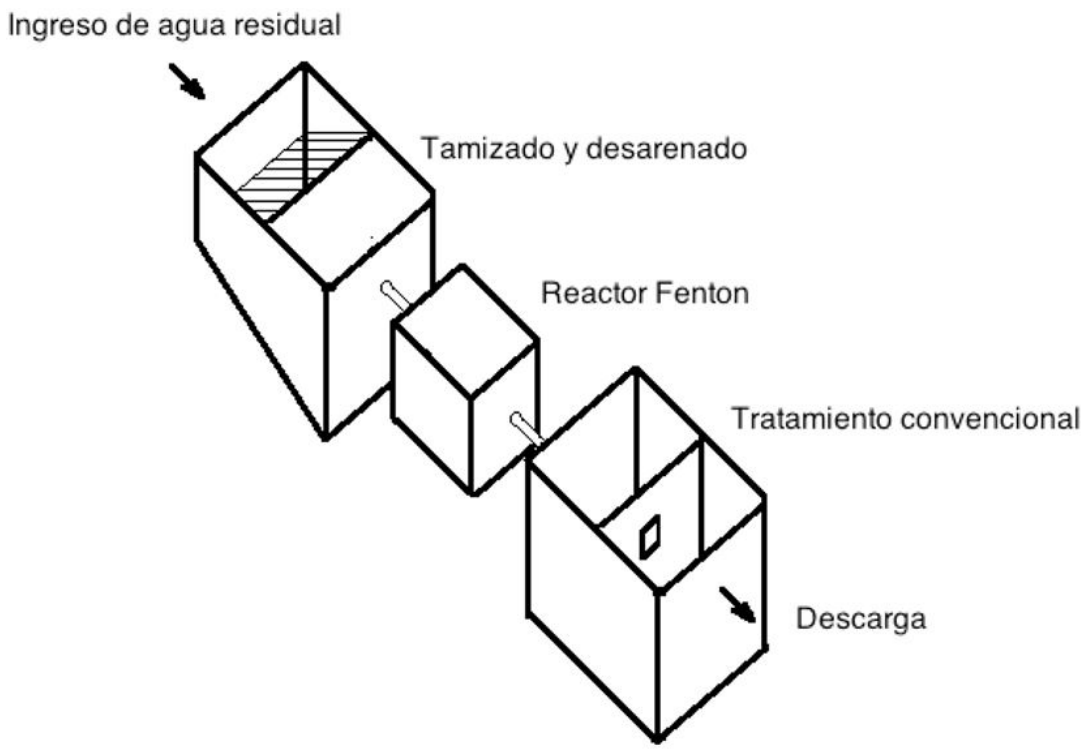

Figura 4. Ubicación del sistema de tratamiento avanzado en una planta de tratamiento de agua residual convencional. 


\section{Conclusiones}

Con los datos presentados se puede concluir que: Las condiciones óptimas para la reacción Fenton fueron $\mathrm{FeSO}_{4} / \mathrm{H}_{2} \mathrm{O}_{2}=1$ y $\mathrm{C}_{6} \mathrm{H}_{8} \mathrm{O}_{7} / \mathrm{H}_{2} \mathrm{O}_{2}=2$; con la colocación de $10 \mathrm{mg} / \mathrm{L}$ de $\mathrm{H}_{2} \mathrm{O}_{2}$. Con estas relaciones la carga orgánica del agua residual se redujo en los parámetros de la $\mathrm{DQO}$ y la $\mathrm{DBO}_{5}$ en eficiencias del $44,4 \%$ y el $12,54 \%$ respectivamente.

Asimismo, se encontró que la reacción Fenton modificado oxidó de manera preferencial a la materia orgánica no biodegradable del agua residual; lo cual aumentó el índice DBO5/DQO de 0,22 a 0,46, y por tanto el agua residual se puede tratar en un sistema convencional.

Finalmente, la implementación de un proceso de tratamiento de oxidación avanzada con Fenton modificado puede ubicarse entre el sistema de tratamiento físico y el sistema de tratamiento biológico, permitiendo prolongar la vida útil de los sistemas de depuración convencionales y con esto mantener la inversión pública desembolsada en construirlos.

\section{Agradecimientos}

Este proyecto es financiado por la Universidad Politécnica Salesiana con sede en Quito- Ecuador, sin este apoyo no hubiera sido posible llevar a buen fin el mismo.

\section{Referencias}

Barbusiński, K y B. Pieczykolan. 2010. “COD Removal From Landfill Leachate Using Fenton Oxidation and Coagulation." Architecture Civil Engineering Environment 3(4):93-100. [en línea] disponible en: https://goo.gl/EVHG6C.

Bautista, P, A. F Mohedano, J. A Casas, J. A Zazo y J. J. Rodriguez. 2008. "An overview of the application of Fenton oxidation to industrial wastewaters treatment." Journal of Chemical Technology E Biotechnology 83(10):13231338. [en línea] doi: https://doi.org/10.1016/ 0048--9697(94)90332--8.

English, A. 2010. Assessment Current Wastewater Treatment and Disposal in Gallatin Country. Gallatin County Planning Board. [en línea] disponible en: https://goo.gl/mFqzZ5.
Furia, T. E. 1973. Handbook of Food Additives. CRC Press. [en línea] disponible en: https://goo.gl/ xHz6f6.

Gil, M, J, A. M Soto, J. I Usma y O. D. Gutiérrez. 2013. "Contaminantes emergentes en aguas, efectos y posibles tratamientos." Producción+ limpia 7(2):52-43. [en línea] disponible en: https:/ /goo. $\mathrm{gl} / \mathrm{pHQd} 36$.

Glaze, W. H, J-W Kang y D. Chapin. 1987. "The Chemistry of Water Treatment Processes Involving Ozone, Hydrogen Peroxide and Ultraviolet Radiation." Ozone: Science \& Engineering 9(4):335-352. [en línea] doi: https://doi.org/10. 1080/01919518708552148.

Isch Zambrano, T. A. 2016. Estudio de un sistema para el tratamiento de efluentes contaminados con compuestos surfactantes por un método Fenton y Fenton Modificado con un agente quelante (EDTA). Tesis de Grado. Escuela Politécnica Nacional, Ecuador. [en línea] disponible en: https://goo.gl/FdcCC4.

Jouravlev, A. 2004. Los servicios de agua potable y saneamiento en el umbral del siglo XXI; recursos naturales e infraestructura. Publicación de las Naciones Unidas. [en línea] disponible en: https:/ /goo.gl/ kEurbJ.

Lentini, E. 2015. El futuro de los servicios de agua de saneamiento en América Latina. Banco Interamericano de Desarrollo, BID. [en línea] disponible en: https://goo.gl/MTX7XK.

Lopes de Morais, J y P. Peralta Zamora. 2005. "Use of advanced oxidation processes to improve the biodegradability of mature landfill leachates." Journal of Hazardous Materials 123(1):181186. [en línea] doi: https://doi.org/10.1016/j. jhazmat.2005.03.041.

Lucho-Constantino, C. A, S. A Medina-Moreno, R. I Beltrán-Hernández, B Juárez-Cruz, G. A Vázquez-Rodríguez y L. Lizárraga-Mendiola. 2015. "Diseño de fosas sépticas rectangulares mediante el uso de la herramienta FOSEP." Revista mexicana de ingeniería química 14:757-765. [en línea] disponible en: https://goo.gl/tvzolu.

MAE. 2015. “Acuerdo Ministerial 061, Tabla 10, del Anexo 1 del Libro VI del TULSMA: Norma de calidad ambiental y de descarga de efluentes al recurso agua." Ministerio del Ambiente, Ecuador. [en línea] disponible en: https:/ /goo.gl/LAed9i. 
Martínez-Huitle, C, A De Battisti, S Ferro, S Reyna, M Cerro y M. Quiroz. 2008. "Removal of the methamidophos pesticide from aqueous solution by electrooxidation using $\mathrm{Pb} / \mathrm{PbO}_{2}, \mathrm{Ti} / \mathrm{SnO}_{2}$ and $\mathrm{Si} / \mathrm{BDD}$ electrodes." Environmental Science and Technology 42(18):6929-6935. [en línea] doi: https://doi.org/10.1021/es8008419.

Mashal, A, J Abu-Daherieh, M Ahmad, Aiouache F y D. Rooney. 2012. Fenton's catalytic oxidation process to treat landfill leachate. In The Sixth Jordan International Chemical Engineering Conference. [en línea] disponible en: https://goo.gl/j4481Y.

Méndez, R, E Chan-Gutiérrez, E Castillo-Borges, E Vázquez-Borges y A. Espadas-Solís. 2012. "Digestión anaerobia de efluentes de fosas sépticas." Ingeniería, Investigación y tecnología 13:339-349. [en línea] disponible en: https://goo.gl/A1xAC9.

Méndez, R, R García, E Castillo y M. Sauri. 2010. "Tratamiento de lixiviados por oxidación Fenton ." Ingeniería e Investigación 30:80-85. [en línea] disponible en: https://goo.gl/Jo5M2p.

Metcalf y Eddy. 2003. Ingeniería de aguas residuales. 4ta ed. New York: McGraw Hill Eduaction.

MTOP. 2010. “Coordinación General de Planificación." Ministerio de Transporte y Obras Públicas, Ecuador. [en línea] disponible en: https://goo. $\mathrm{gl} / \mathrm{zUQkhf}$.

Ortiz de Zárate, J. M y L. A. Aguila Apodaca. 1997. "La depuración biológica de efluentes industriales: centro de tratamiento de residuos industriales de Galicia." Ingeniería Química (336):147-153. [en línea] disponible en: https://goo.gl/atqsFk.

Oviedo, C y J. Rodríguez. 2003. "EDTA: the chelating agent under environmental scrutiny." Quimica Nova 26(6):901-905. [en línea] doi: http:/ /dx. doi.org/10.1590/S0100--40422003000600020.
Pawar, V y S. Gawande. 2015. "An overview of the Fenton Process for Industrial Wastewater." IOSR-JMCE pp. 127-136. [en línea] disponible en: https://goo.gl/8Num3F.

Pawlowski, L. 1994. "Standard methods for the examination of water and wastewater,." Science of The Total Environment 142(3):227228. [en línea] doi: https://doi.org/10.1016/ 0048--9697(94)90332--8.

REPAMAR. 1998. Impacto ambiental de productos químicos auxiliares usados en la industrial textil Argentina. Red Panamericana de Manejo Ambiental de Residuos. Informe Final. [en línea] disponible en: https://goo.gl/gDETSz.

Seol, Y y Javandel. I. 2008. "Citric acid-modified Fenton's reaction for the oxidation of chlorinated ethylenes in soil solution systems." Chemosphere 72(4):537-542. [en línea] doi: https://doi.org/10. 1016/j.chemosphere.2008.03.052.

Somich, C, M Muldoon y P. Kearney. 1990. “Onsite treatment of pesticide waste and rinsate using ozone and biologically active soil." Environmental Science E Technology 24(5):745-749. [en línea] doi: https://doi.org/10.1021/es00075a021.

UNESCO. 2017. “ ¿Son las aguas residuales el nuevo "oro negro"?" Presentación pública del Informe de las Naciones Unidas sobre el Desarrollo de los Recursos Hídricos. [en línea] disponible en: https://goo.gl/3BmScu.

Zazouli, M. A, Z Yousefi, A Eslami y M. B. Ardebilian. 2012. "Municipal solid waste landfill leachate treatment by fenton, photo-fenton and fenton-like processes: Effect of some variables." Iranian Journal of Environmental Health Science $\mathcal{E}$ Engineering 9(1):3. [en línea] doi: https:/ / doi.org/ 10.1186/1735--2746--9--3. 